denominator in 'paramedic attendance'

$(48 / 119) \times 100 \%=40.3 \%$ as stated?

The authors conclude that $\mathrm{BDH}$ has a robust emergency protocol and recordkeeping system. How can a recordkeeping system with an incomplete record rate of $20 \%$ $(24 / 119)$ ever be called robust?

P. Nederlof, Evesham

The authors, S. Sooch, A. Kaur and B. Ahmed respond: We thank your reader for their comments. We need to acknowledge that the Datix system is a web-based online incidence reporting software usually completed by the dentist but in some cases by another member of the dental team. As investigators we access these data from the spreadsheet held by the governance manager. To our knowledge, this is the only way we can capture medical emergencies as reported by the dental team for our hospital. The Datix online reporting involves free text data input by the person filling in the report. This explains some of the discrepancies as some of the data are not documented and therefore we need to account for this. In saying this, we were stating that the nature of the medical emergency was known in 119 cases, however any additional demographic information (ie age) had been missing from some of the data entries; therefore the bar graph depicted a total of 95 . Hence why Figure 1, which showed age groups of people affected and Table 1, which shows the frequency of medical emergencies, add up to different figures, as we were trying to depict these separately.

We excluded the four cases from 119 to give us a denominator of 115 (not from 95 to 91), as the dataset again was incomplete and we decided to continue with the 115 definitive cases and outcomes available.

We used the denominator 119 for paramedics called to the hospital, as the data were always available for each case regarding paramedic attendance as demonstrated in Figure 2.

Patient safety is a core value of our hospital; we like many other hospitals use the Datix system as a platform in recording untoward events such as medical emergencies. We accept the Datix system could be improved as it relies heavily on the information input by the reporters: missing data, a wrong clinical diagnosis input in the system can give rise to discrepancies; however, changing the system was beyond the scope of our project.

DOI: $10.1038 /$ sj.bdj.2018.366

\section{Editorial decisions}

\section{Outraged}

Sir, I have been an avid reader of the $B D J$ since I was a first year dental student and I have never felt so compelled to write to you and express my outrage after reading the first two letters from Volume 224 No. 4, 23 February 2018, under the subheading 'Editorial decisions.',

I would like you to know that I loved the cartoon cover arts, especially the one with the mouse and the fox. I always enjoy the different types of artwork on the $B D J$ and reading about their background.

As for the Christmas edition I simply cannot fathom why anyone would be against something light hearted and humorous. Dentistry is a tough profession and I am sure most dentists have felt down, depressed and anxious at some point. I personally feel the festive season can be quite overwhelming as there is a pressure on people in general to be all happy and jolly, but for us the same pressures still apply; indeed it can be an even busier time of year with extra patients. Reading something funny written by your fellow professionals could make the world of difference for someone feeling a bit low. It could bring a smile and some cheer for a dentist feeling overwhelmed.

I write this letter to assure you that the majority of the profession appreciate and enjoy the more light-hearted elements of the journal and request that this tradition continues. Yes dentistry is a serious profession, and yes we all enjoy reading the factual content of the journal, but having something fun certainly doesn't take any credit away from this well respected journal. Indeed it is of my opinion it actually makes it even more loved as the journal which is known to have something for every dentist no matter what discipline they are in.

N. Jamil, Bury, Lancashire

1. Sperber $\mathrm{G}$. Editorial decisions: Diminishing credibility. $\mathrm{Br}$ Dent J 2018; 224: 197.

2. Lawrence A J. Pathetic jokes. Br Dent J 2018; 224: 197 198.

DOI: 10.1038/sj.bdj.2018.367

\section{Grave concern}

Sir, I am writing to you to express my grave concern regarding a recent entry for Zollipops in the British Dental Journal ${ }^{1}$ under the 'Dental products and services' section.

Whilst I fully appreciate that the $B D J$ has a disclaimer at the top of the page indicating that the service does not imply endorsement by the $B D J$, many dentists do not read this and would assume that because this has been published in a professional journal this product has some value.

The advert is basically for a sugar free lollipop - my concerns relate particularly to the logos being used to promote the product ie 'The clean teeth pops' and 'The after you eat treat for a healthy smile'.

Implying that eating a lollipop, regardless of when you eat it can somehow 'clean' your teeth is a very misleading message for the general public.

As health professionals it is imperative that we all give consistent, evidence-based messages to the general public. The statement that 'Stein Foods ... wants dentists to stand behind and promote the brand as a healthy and good way for parents to look after their children's teeth' really concerned me. Although sugar-free sweets may not directly damage teeth (some brands however can be acidic and could potentially be implicated in tooth erosion) regular consumption could help encourage a 'sweet tooth' which could certainly have implications for general health.

I do feel that the British Dental Journal is better than this and should be more proactive in filtering out unsuitable adverts before publication.

J. Thomas, Swansea

1. Product News. A lollipop that's good for your teeth? Br Dent J 2018; 224: 118.

DOI: $10.1038 /$ sj.bdj.2018.368

\section{Dental careers}

\section{To specialise or not?}

Sir, I am writing to you as a recently qualified and aspiring dentist. Although I am in my early years, I find myself, like many of my other dental colleagues, at a career crossroads. With so many specialities and sub-specialities on offer as a dentist, it can often be confusing what to choose. I hear from many dentists that you don't need to specialise to excel within a sub-speciality. However, I hear from just as many that to excel and reach the pinnacle as a dentist, you should aim to specialise.

Does a dentist on the specialist register hold greater value and worth than one that isn't? Alternatively, will having an MSc, which has been obtained over a two-year period part time, put you in just as much demand as a dentist who has spent the last three years, working full time, on the specialist register?

With so many different courses on offer, it can often prove difficult to decide which one 